\title{
Article \\ A Full-Period Mathematical Model for a Hybrid-Rotor Bearingless Switched Reluctance Motor
}

\author{
Zeyuan Liu ${ }^{1,2, * \mathbb{D}}$, Mei Chen ${ }^{1}$, Yan Yang ${ }^{1}$, Chengzi Liu ${ }^{1}$ and Hui Gao ${ }^{1}$ \\ 1 College of Automation \& College of Artificial Intelligence, Nanjing University of Posts and \\ Telecommunications, Nanjing 210023, China; cm15250998376@sina.com (M.C.); \\ yangyan0405@njupt.edu.cn (Y.Y.); liuchengzi@njupt.edu.cn (C.L.); gaoh@njupt.edu.cn (H.G.) \\ 2 Key Laboratory of Special Machine and High Voltage Apparatus, Shenyang University of Technology, \\ Ministry of Education, Shenyang 110870, China \\ * Correspondence: liuzy@njupt.edu.cn
}

Citation: Liu, Z.; Chen, M.; Yang, Y.; Liu, C.; Gao, H. A Full-Period

Mathematical Model for a

Hybrid-Rotor Bearingless Switched Reluctance Motor. Symmetry 2021, 13, 2383. https://doi.org/10.3390/ sym13122383

Academic Editors: Wenliang Zhao, Dezhi Chen and Jinhua Chen

Received: 15 November 2021 Accepted: 8 December 2021 Published: 10 December 2021

Publisher's Note: MDPI stays neutral with regard to jurisdictional claims in published maps and institutional affiliations.

Copyright: (c) 2021 by the authors. Licensee MDPI, Basel, Switzerland. This article is an open access article distributed under the terms and conditions of the Creative Commons Attribution (CC BY) license (https:// creativecommons.org/licenses/by/ $4.0 /)$.

\begin{abstract}
A bearingless switched reluctance motor (BSRM) has the combined characteristics of a switched reluctance motor (SRM) and a magnetic bearing. The hybrid-rotor BSRM (HBSRM) discussed in the paper has a twelve-pole stator and an eight-pole hybrid rotor, which is composed of a cylindrical rotor and a salient-pole rotor. Although the asymmetry of the hybrid rotor makes the structure and magnetic field of the HBSRM more complex, it can always produce a significant amount of magnetic pulling force to levitate a rotor shaft at all the rotor angular positions of each phase, which is not available in a traditional BSRM. The classical mathematical model for a conventional BSRM is valid only when its rotor rotates from the start of the overlap position to the aligned position, and the radial force and torque derived from this model are discontinuous at the aligned positon, which is harmful to the motor's stable operation. In this paper, a full-period mathematical model on the assumption that the gap permeance is cut apart by straight lines or improved elliptical lines for a 12/8-pole HBSRM is provided. On the basis of this mathematical model, the continuity of the radial force and torque at all the rotor angular positions can be guaranteed, and the fine characteristics of this mathematical model have been verified by simulations.
\end{abstract}

Keywords: bearingless switched reluctance motor; mathematical model; radial force; torque

\section{Introduction}

Switched reluctance motor (SRM) has superior performance under special environments such as the starter/generator in all electric/multi-electric aeroengine, vacuum pump, and flywheel energy storage systems, because of its simple structure, low cost, robustness, and suitability for operation in high temperatures [1-3]. The SRM generally has a short air gap length in order to effectively produce the rotational reluctance torque, and a considerable magnetic attraction force is also simultaneously generated in radial directions. It is very convenient to utilize this radial force so as to levitate a rotor shaft. Therefore, the SRM has a good feature as a bearingless motor, which is characterized by the integration of an electrical motor and magnetic bearing [4-6].

A bearingless switched reluctance motor (BSRM) with differential stator-winding configuration was proposed in References [7-10]. This structure has two kinds of stator windings composed of motor main windings and radial force windings in the same stator in order to produce the required radial force that may realize the suspension of the rotor shaft without mechanical contacts or lubrication. Then, some single-winding BSRMs with the same structures as traditional SRM have also been developed [11-14]. In these structures, the required radial force and rotational torque can be simultaneously produced by independently controlling currents in all the windings.

In a control system of BSRM, fast current regulation is required for radial force and torque control. Especially for continuous magnetic levitation, suspended forces must be 
controlled at all rotor angular positions. Therefore, an accurate mathematical model for a BSRM is very essential for stable suspension and rotation. Theoretical formulas of radial force and torque have been derived from a magnetic equivalent circuit of the 12/8-pole BSRM $[7,8]$. In these models, the gap permeance has been cut apart by straight lines and elliptical lines for the BSRM. For the derivation of the gap permeance in fringing fluxes, the magnetic paths are assumed to be an elliptical shape, and the variable " $k$ " is used to represent the shape of the elliptical lines. In References $[7,8]$, the variable $k$ is set to a constant to calculate the shape of the ellipse. However, in fact, this ellipse coefficient $k$ is a variable and decided by the rotor angular positions and air gap length. Therefore, when the rotor is at the aligned position, the torque of BSRM derived from this method is not zero, and the radial force is also discontinuous. Finally, it is difficult to stably control the suspension and rotation of the rotor at the same time with this classical model.

In the traditional BSRM, if stator and rotor teeth are in aligned positions, the radial force can be effectively established; but it is very small when the rotor rotates from the unaligned position to the start of the overlap position. Therefore, the existing models are derived only when the rotor varies from the start of the overlap position to the aligned position of each phase. When the BSRM operates as a motor, the currents in each phase winding are expected to be completely controlled during the rising range of the inductance. Therefore, the excitation interval of each phase is only set between the start of the overlap position and the aligned position. However, this excitation mode is unfavorable to the torque output, especially when the motor speed is high [15]. The specific reasons are as follows: The phase current cannot reach the given value quickly due to the bigger increase rate of inductance at the start of the overlap position between the stator and rotor poles, so the turn-on angle of each phase for a traditional SRM is usually set in the unaligned position or in the range of the inductance drop. On the other hand, the torque ripple increases, because the freewheeling current causes the motor to operate in the negative torque range; thus, the excitation phase of a traditional SRM is usually turned off before the aligned position, and the higher the motor speed, the farther the turn-off angle is from this aligned position. Therefore, for a BSRM system, it is necessary to improve the magnetic levitation force near the unaligned positions in the structure and optimize each phase conduction region in the control.

In this paper, a hybrid-rotor BSRM (HBSRM) with a twelve-pole stator and an eightpole hybrid rotor is discussed. Its hybrid rotor is composed of circular and scalloped lamination segments, and the cylindrical rotor part is used to generate additional radial forces in order to suspend the shaft near the unaligned positions. It is essential to derive the novel mathematical model of the radial force and torque applicable to all rotor positions. Due to the asymmetry of the hybrid rotor, the magnetic field distribution of HBSRM becomes more complex. Therefore, it is more difficult to deduce the full-period mathematical model of HBSRM, especially when the rotor is between the completely misaligned position and the start of the overlap position.

The arrangement of this paper is as follows: Firstly, the structure and operating principle of a 12/8-pole HBSRM are briefly introduced. Secondly, in order to simplify the magnetic circuit analysis and deduce the mathematical model conveniently, the 12/8pole HBSRM is equivalent to two parts, namely a 12/8-pole BSRM and a 12-pole active magnetic bearing (AMB). The torque expressions are derived from the BSRM part, and the radial force formulas are obtained by superposition of both the BSRM and AMB parts. Finally, the fine characteristics of this full-period mathematical model have been verified by simulations.

\section{Structure and Working Principle}

The HBSRM with a twelve-pole stator and an eight-pole hybrid rotor is discussed in this paper, and its hybrid rotor is composed of a cylindrical rotor and a salient pole rotor, as shown in Figure 1. Same as the traditional 12/8-pole SRM, each stator tooth in this motor still has a coil, and the four sets of coils separated by $90^{\circ}$ in space form one 
phase. Therefore, twelve sets of coils are divided into three phases: A-phase, B-phase, and C-phase, and each phase is separated by $30^{\circ}$ in space. Three-phase windings are excited in turn every $15^{\circ}$, and the rotor rotates counterclockwise. In the paper, $\theta$ indicates the rotor angular position, and $\theta=0$ is defined as the aligned position of the A-phase. In Figure 2, the rotor angular $\theta$ of is $-\pi / 24\left(-7.5^{\circ}\right)$.

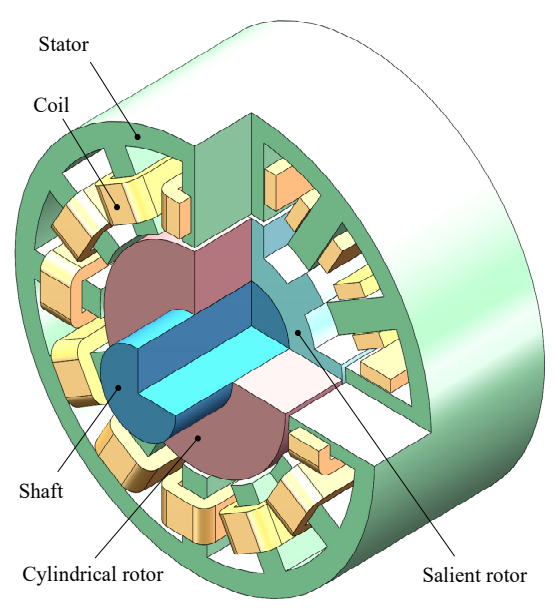

Figure 1. Three-dimensional schematic of the 12/8-pole HBSRM.

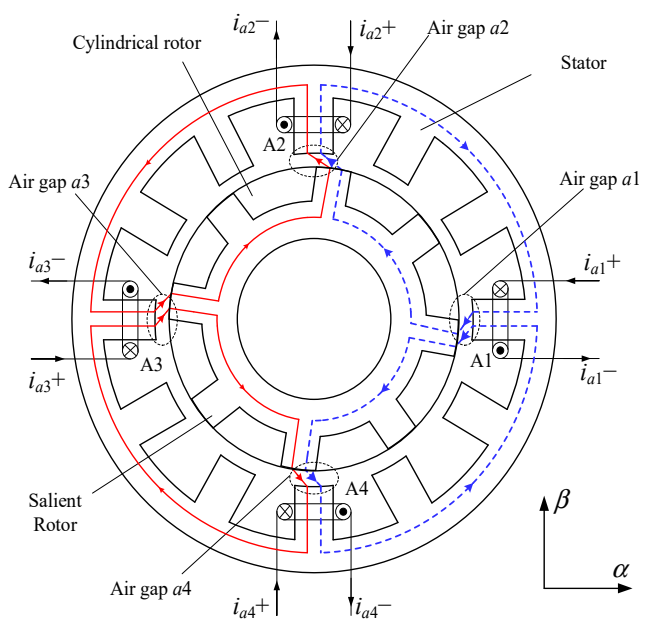

Figure 2. A-phase winding configuration and the principle of radial force production.

In Figure 2, the blue broken lines show the two-pole fluxes produced by the current $i_{a 1}$ in the winding A1, and the red solid lines show the two-pole fluxes produced by the current $i_{a 3}$ in the winding A3. If $i_{a 1}>i_{a 3}$, the flux density in the air gap $a 1$ is greater than that in the air gap $a 3$. These two unequal magnetic fluxes result in the radial force $F_{\alpha}$ acting on the rotor shaft toward the positive direction on the $\alpha$-axis. On the other hand, when $i_{a 1}<i_{a 3}$, the flux in the air gap $a 1$ is less than that in the air gap $a 3$, and then, a negative radial force on the $\alpha$-axis direction may be generated. Moreover, a radial force $F_{\beta}$ on the $\beta$-axis direction can be produced by controlling the currents $i_{a 2}$ in the winding A2 and $i_{a 4}$ in the winding A4. Therefore, the desired magnetic levitation force can be produced in a radial direction. Similarly, this principle of radial force production can be applied to the B- and C-phase. The radial force can be successfully built by these three phases in turn every $15^{\circ}$.

Figure 3 shows the distribution of the phase inductance, radial force, and torque in the two types of bearingless motor structures; $L_{\mathrm{HBSRM}}$ is the phase inductance of the $12 / 8$ pole HBSRM, and $L_{\mathrm{BSRM}}$ is the phase inductance of the 12/8-pole BSRM. In a 12/8-pole BSRM, when its stator tooth and rotor tooth are in a completely misaligned positions or 
the overlapping area is very small, the rotor cannot suspend, or requires a large current to suspend, the shaft. Thus, there may be a dead zone of levitation in each rotor period of the traditional BSRM, such as regions I and IV, as shown in Figure 3. However, for a 12/8-pole HBSRM, the magnetic pulling force between the stator and cylindrical rotor is enough to suspend the shaft, so the levitation of its rotor shaft can be achieved in all the rotor angular positions.

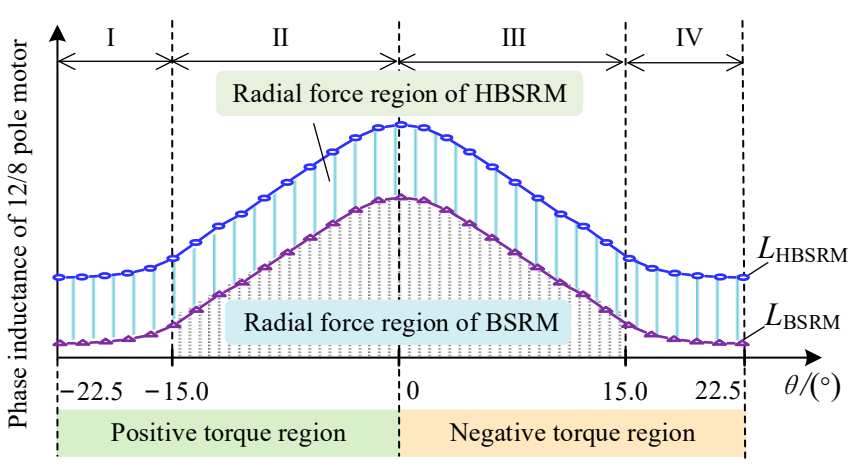

Figure 3. Distribution of the inductance, radial force, and torque of a 12/8-pole BSRM and HBSRM.

In view of the radial force characteristics of the 12/8-pole HBSRM, its excitation range of each phase is redesigned to be the same as that of a conventional SRM, as shown in Figure 4. Taking the A-phase of a 12/8-pole HBSRM as an example, it can be excited from $-22.5^{\circ}$ to $-7.5^{\circ}$. For the A-phase of a 12/8-pole BSRM, its excitation range is generally designed as $\left(-15^{\circ}, 0\right)$. It can be seen from Figure 4 , compared with the traditional BSRM, that the switching angle of each phase of the 12/8-pole HBSRM moves forward by $7.5^{\circ}$. This excitation mode has the following two advantages: (a) the current tracking effect at the opening time can be improved due to the smaller change rate of the phase inductance, and (b) the torque ripple can be reduced at a high speed, because the motor running in the negative torque region is avoided. In Figure $4, L_{a}, L_{b}$, and $L_{c}$ are the inductance of the A-, B-, and C-phases of a 12/8-pole HBSRM, and $i_{a 1} \sim i_{a 4}, i_{b 1} \sim i_{b 4}$, and $i_{c 1} \sim i_{c 4}$ are the four winding currents of the A-, B-, and C-phases.

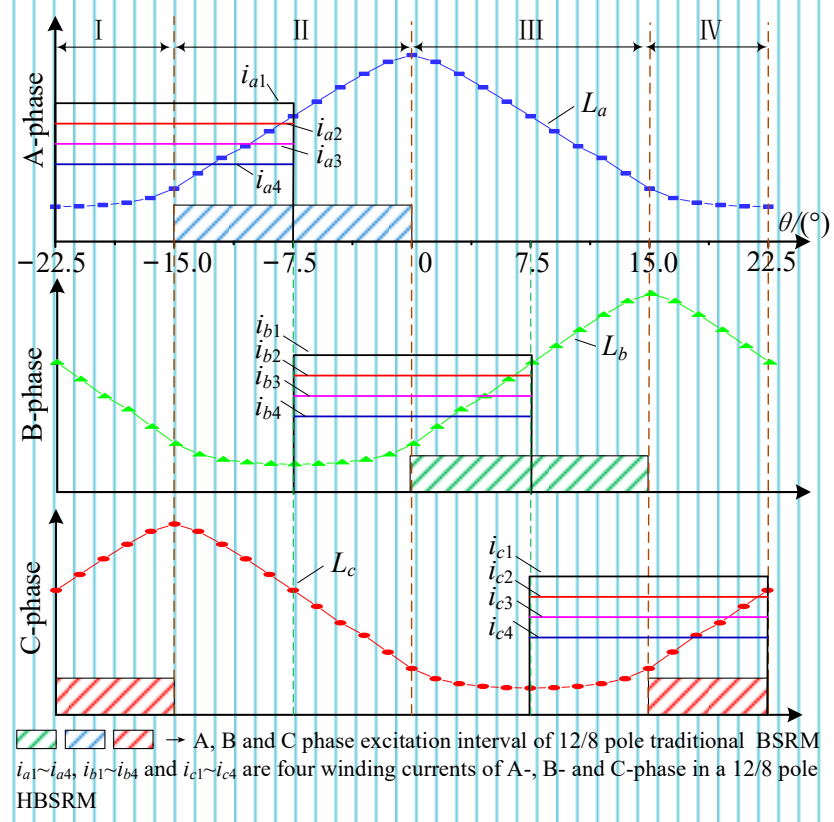

Figure 4. Three-phase excitation ranges of a 12/8-pole BSRM and HBSRM. 


\section{Mathematical Model}

For the HBSRM discussed in the paper, the asymmetry of the hybrid-rotor structure makes its magnetic field be distributed asymmetrically. In order to simplify the magnetic circuit analysis and deduce the mathematical model conveniently, the 12/8-pole HBSRM is equivalent to two parts, namely a 12/8-pole BSRM and a 12-pole AMB. Moreover, the three-dimensional magnetic field of HBSRM can also be regarded as two two-dimensional fields. The magnetic fluxes of two equivalent parts produced by the A-phase current $i_{a 1} \sim i_{a 4}$ are as shown in Figure 5. The dimensions of a prototype are shown in Table 1.

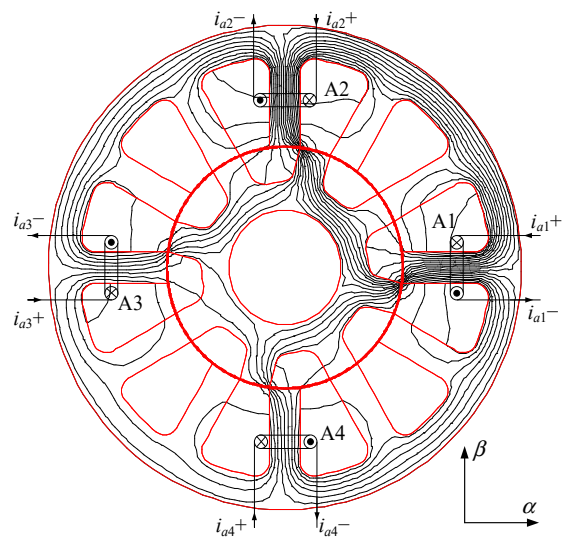

(a)

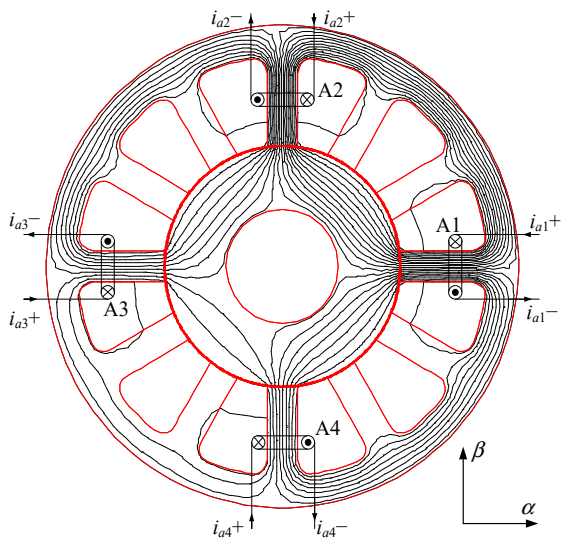

(b)

Figure 5. Magnetic flux distribution of two equivalent parts: (a) BSRM part and (b) AMB part.

Table 1. Parameters of the prototype.

\begin{tabular}{cc}
\hline Parameter & Value \\
\hline Outer diameter of stator & $105.0 \mathrm{~mm}$ \\
Inner diameter of stator & $52.7 \mathrm{~mm}$ \\
Stator yoke & $6.9 \mathrm{~mm}$ \\
Outer diameter of salient rotor & $52.0 \mathrm{~mm}$ \\
Salient rotor yoke & $6.0 \mathrm{~mm}$ \\
Shaft diameter & $25.0 \mathrm{~mm}$ \\
Stator pole arc angle & $15^{\circ}$ \\
Salient rotor pole arc angle & $15^{\circ}$ \\
Number of stator poles & 12 \\
Number of salient rotor poles & 8 \\
Number of winding turns & 60 \\
Number of parallel winding turns & 2 \\
Length of salient rotor stack & $75.0 \mathrm{~mm}$ \\
Length of cylindrical rotor stack & $35.0 \mathrm{~mm}$ \\
\hline
\end{tabular}

\subsection{Derivation of Inductances}

Figure 6 shows the magnetic equivalent circuit of the 12/8-pole HBSRM. The voltage sources indicate magnetomotive forces of these twelve windings, and the air gap permeances are expressed as equivalent resistances. The magnetic equivalent circuit of the A-phase is shown in Figure 7, where $N$ represents the number of turns of each winding in each stator tooth, $P_{a 1} \sim P_{a 4}$ show the air gap permeances of the A-phase stator teeth, and $\Phi_{a 1} \sim \Phi_{a 4}$ indicate the magnetic fluxes of each stator tooth. Only the magnetic equivalent circuit of the A-phase is used to make a simple calculation because of a little mutual inductance among the A-, B-, and C-phases in a traditional SRM. 


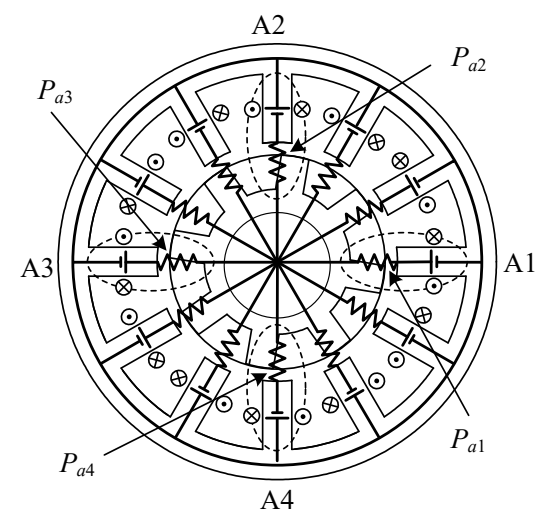

Figure 6. Magnetic equivalent circuit of the 12/8-pole HBSRM.

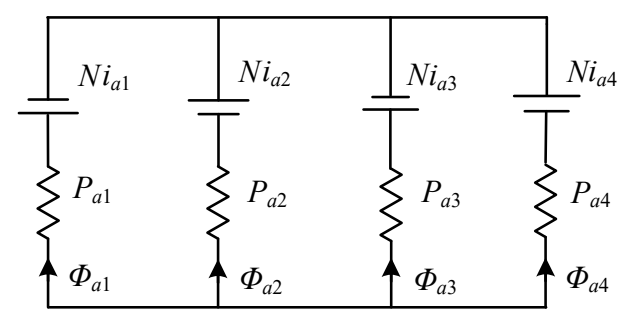

Figure 7. Magnetic equivalent circuit of the A-phase.

If each permeance shown as $P_{a 1} \sim P_{a 4}$ can simply be assumed, the self and mutual inductances of each winding of the A-phase can be calculated from Figure 7. The detailed derivations are similar to that of the 12/8-pole double-winding BSRM in References [7,8]. The self-inductances and the mutual inductances are expressed as

$$
\left\{\begin{array}{l}
L_{a 1}=\frac{N^{2}}{P} P_{a 1}\left(P_{a 2}+P_{a 3}+P_{a 4}\right), L_{a 2}=\frac{N^{2}}{P} P_{a 2}\left(P_{a 1}+P_{a 3}+P_{a 4}\right), \\
L_{a 3}=\frac{N^{2}}{P} P_{a 3}\left(P_{a 1}+P_{a 2}+P_{a 4}\right), L_{a 4}=\frac{N^{2}}{P} P_{a 4}\left(P_{a 1}+P_{a 2}+P_{a 3}\right), \\
M_{a 12}=\frac{N^{2}}{P} P_{a 1} P_{a 2}, M_{a 13}=-\frac{N^{2}}{P} P_{a 1} P_{a 3}, M_{a 14}=\frac{N^{2}}{P} P_{a 1} P_{a 4}, \\
M_{a 23}=\frac{N^{2}}{P} P_{a 2} P_{a 3}, M_{a 24}=-\frac{N^{2}}{P} P_{a 2} P_{a 4}, M_{a 34}=\frac{N^{2}}{P} P_{a 3} P_{a 4}
\end{array}\right.
$$

where $L_{a 1} \sim L_{a 4}$ are the self-inductances of the windings $\mathrm{A} 1 \sim \mathrm{A} 4, M_{a 12}$ is the mutual inductance between $\mathrm{A} 1$ and $\mathrm{A} 2, M_{a 13}$ is the mutual inductance between $\mathrm{A} 1$ and $\mathrm{A} 3, M_{a 14}$ is the mutual inductance between $\mathrm{A} 1$ and $\mathrm{A} 4, M_{a 23}$ is the mutual inductance between $\mathrm{A} 2$ and $\mathrm{A} 3$, $M_{a 24}$ is the mutual inductance between $\mathrm{A} 2$ and $\mathrm{A} 4, M_{a 34}$ is the mutual inductance between $\mathrm{A} 3$ and $\mathrm{A} 4$, and $P$ is the sum of the permeances $P_{a 1} \sim P_{a 4}$.

\subsection{Radial Forces in the BSRM Part}

3.2.1. Calculation of the Permeances

(a) $0 \leq|\theta| \leq \frac{\pi}{12}$

As shown in Figure 8, the permeance $P_{a 1}$ of the air gap $a 1$ is divided into three parts of permeances: $P_{1} \sim P_{3}$. In this paper, $P_{1}$ is derived by using a straight magnetic path, and $P_{2}$ and $P_{3}$ are calculated by the assumed magnetic paths. In Figure $8, t$ represents an angular position on a rotor circular surface, $d t$ is a derivative of $t, l_{g}$ is the air gap length after the rotor eccentricity, $l_{0}$ is the average air gap length without the rotor eccentricity, $\alpha$ is the radial displacement of the rotor in the $\alpha$-axis direction, $r$ is the rotor radius, and $d P_{2}$ and $d P_{3}$ are the permeances of the infinitesimal width of the assumed magnetic path. 


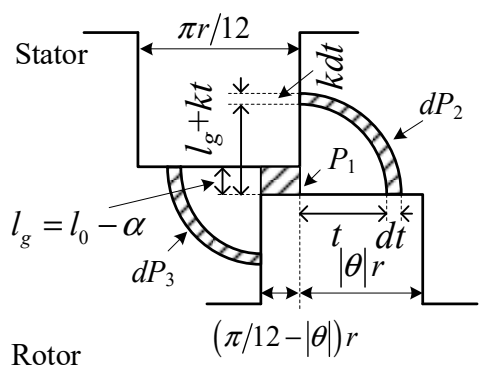

Figure 8. Assumed magnetic paths of air gap $a 1$ at the positons of $0 \leq|\theta| \leq \frac{\pi}{12}$ in the BSRM part.

The assumed magnetic paths are composed of improved elliptical lines using a variable $k$. The finite element analysis of an electromagnetic field in the literature [16] showed that it relies on the rotor angular position $\theta$, the rotor radius $r$, the average air gap length $l_{0}$, and the variable $a$. Reference [16] used the least squares method to calculate the variable $a$ from the finite element analysis. In the paper, in order to simplify the derivation and computation, the coefficient $k$ is directly simplified as

$$
k=\frac{|\theta| r}{l_{0}+|\theta| r}
$$

According to the definition of permeance, $d P_{2}$ can be written as

$$
d P_{2}=\frac{\mu_{0} \times d s}{l}
$$

where $\mu_{0}$ is permeability in the air, $s$ represents the cross-sectional area of $d P_{2}, d s$ is a derivative of $s$, and $l$ is the average length of the magnetic path.

As shown in Figure 8, the short half-axis of the elliptical line is $t$, and the long half-axis may be written as $l_{0}+k t$. Correspondingly, the average cross-sectional area $d s$ of $d P_{2}$ can be approximately expressed as

$$
d s \approx \frac{h_{s}(d t+k d t)}{2}=\frac{h_{s}(k+1) \times d t}{2}
$$

where $h_{s}$ is the stack length of the scalloped lamination segments.

The average length of the assumed magnetic path is approximately expressed as

$$
l \approx l_{g}+\frac{\pi}{2} t=l_{0}-\alpha+\frac{\pi}{2} t
$$

Substituting Equations (2), (4), and (5) into Equation (3) yields

$$
d P_{2}=\left(\frac{l_{0}+2|\theta| r}{l_{0}+|\theta| r}\right) \frac{\mu_{0} h_{s} \times d t}{2\left(l_{0}-\alpha\right)+\pi t}
$$

Moreover, the permeance $P_{2}$ can be calculated as

$$
P_{2}=\int_{0}^{r|\theta|} d P_{2}=\frac{\mu_{0} h_{s}}{\pi}\left(\frac{l_{0}+2|\theta| r}{l_{0}+|\theta| r}\right) \ln \left(\frac{2 l_{0}^{2}+\pi r|\theta|\left(l_{0}+\alpha\right)}{2 l_{0}^{2}}\right)
$$

Similarly, the permeance $P_{3}$ is derived considering the symmetrical fringing flux paths as

$$
P_{3}=\frac{\mu_{0} h_{s}}{\pi}\left(\frac{l_{0}+2|\theta| r}{l_{0}+|\theta| r}\right) \ln \left(\frac{2 l_{0}^{2}+\pi r|\theta|\left(l_{0}+\alpha\right)}{2 l_{0}^{2}}\right)
$$


The permeance $P_{1}$ of the air gap between the rotor and stator poles is expressed as

$$
P_{1}=\frac{\mu_{0} h_{s} r\left(\frac{\pi}{12}-|\theta|\right)\left(l_{0}+\alpha\right)}{l_{0}^{2}}
$$

Moreover, the total permeances $P_{a 1}$ can be obtained by summing $P_{1}, P_{2}$, and $P_{3}$ as

$$
P_{a 1}=P_{1}+P_{2}+P_{3}=\frac{\mu_{0} h_{s} r\left(\frac{\pi}{12}-|\theta|\right)\left(l_{0}+\alpha\right)}{l_{0}^{2}}+\frac{2 \mu_{0} h_{s}}{\pi}\left(\frac{l_{0}+2|\theta| r}{l_{0}+|\theta| r}\right) \ln \left(\frac{2 l_{0}^{2}+\pi r|\theta|\left(l_{0}+\alpha\right)}{2 l_{0}^{2}}\right)
$$

Similarly, $P_{a 2}, P_{a 3}$, and $P_{a 4}$ at the positions of $0 \leq|\theta| \leq \frac{\pi}{12}$ can be derived by using the above method.

(b) $\frac{\pi}{12} \leq|\theta| \leq \frac{\pi}{8}$

Figure 9 shows the enlarged magnetic flux of the BSRM part at the unaligned position obtained by a two-dimensional finite element analysis. The permeance $P_{a 1}$ of the air gap $a 1$ is composed of four parts of permeances: $P_{4} \sim P_{7}$. Similar to the assumed magnetic path at the positions of $0 \leq|\theta| \leq \frac{\pi}{12}$, as shown in Figure 8, the shape of the fringing magnetic paths is still set in the shape of an ellipse. When $\frac{\pi}{12} \leq|\theta| \leq \frac{\pi}{8}$, the assumed magnetic paths of the air gap $a 1$ in the BSRM part are shown in Figure 10. $d P_{4}$ and $d P_{5}$ are the permeances of the infinitesimal width of the assumed magnetic path.

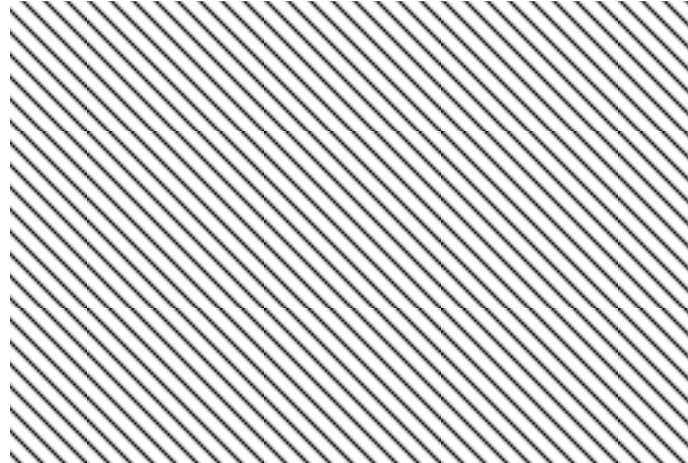

Figure 9. Magnetic fluxes analyzed with the finite element method at the unaligned position in the BSRM part.

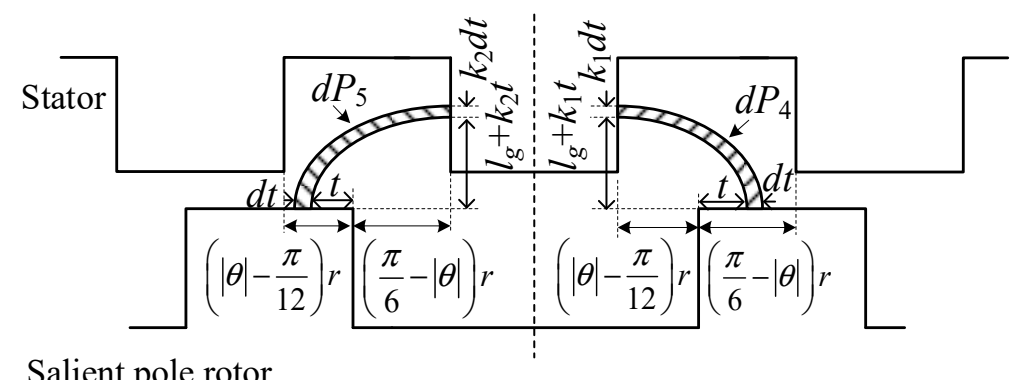

Salient pole rotor

Figure 10. Assumed magnetic paths of the air gap $a 1$ at the positons of $\frac{\pi}{12} \leq|\theta| \leq \frac{\pi}{8}$ in the BSRM part.

Considering the symmetry of the fringing magnetic paths, the total permeances $P_{a 1}$ at the positions of $\frac{\pi}{12} \leq|\theta| \leq \frac{\pi}{8}$ can be written as

$$
P_{a 1}=2 P_{4}+2 P_{5}
$$


For the part of permeance $P_{4}$, we can get the following expressions:

$$
\left\{\begin{array}{l}
l_{1} \approx l_{0}-\alpha+\frac{\pi}{2}\left(|\theta|-\frac{\pi}{12}\right) r+\frac{\pi}{2} t \\
d s_{1}=\frac{h_{s}\left(k_{1}+1\right) d t}{2} \\
k_{1}=\frac{\left(|\theta|-\frac{\pi}{12}\right) r}{l_{0}+\left(|\theta|-\frac{\pi}{12}\right) r} \\
d P_{4}=\frac{\mu_{0} \times d s_{1}}{l_{1}}
\end{array}\right.
$$

where $l_{1}$ is the average length of the equivalent magnetic path, $s_{1}$ is the cross-sectional area of $d P_{4}, d s_{1}$ is a derivative of $s_{1}$, and the variable $k_{1}$ is used to determine the shape of the ellipse at the positions of $\frac{\pi}{12} \leq|\theta| \leq \frac{\pi}{8}$.

Moreover, the permeance $P_{4}$ may be obtained as

$$
P_{4}=\int_{0}^{\left(\frac{\pi}{6}-|\theta|\right) r} d P_{4}=\frac{\mu_{0} h_{s}}{\pi}\left(\frac{l_{0}+2\left(|\theta|-\frac{\pi}{12}\right) r}{l_{0}+\left(|\theta|-\frac{\pi}{12}\right) r}\right) \ln \left(\frac{2 l_{0}^{2}+\frac{\pi^{2}}{12} r\left(l_{0}+\alpha\right)}{2 l_{0}^{2}+\pi r\left(|\theta|-\frac{\pi}{12}\right)\left(l_{0}+\alpha\right)}\right)
$$

Similarly, for the part of permeance $P_{5}$, some related expressions can be written as

$$
\left\{\begin{array}{l}
l_{2} \approx l_{0}-\alpha+\frac{\pi}{2}\left(\frac{\pi}{6}-|\theta|\right) r+\frac{\pi}{2} t \\
d s_{2}=\frac{h_{s}\left(k_{2}+1\right) d t}{2} \\
k_{2}=\frac{\left(\frac{\pi}{6}-|\theta|\right) r}{l_{0}+\left(\frac{\pi}{6}-|\theta|\right) r} \\
d P_{5}=\frac{\mu_{0} \times d s_{2}}{l_{2}}
\end{array}\right.
$$

where $l_{2}$ is the average length of the assumed magnetic path, $s_{2}$ is the cross-sectional area of $d P_{5}, d s_{2}$ is a derivative of $s_{2}$, and the variable $k_{2}$ is used for calculating the shape of the ellipse at the positions of $\frac{\pi}{12} \leq|\theta| \leq \frac{\pi}{8}$.

Then, the total permeance $P_{5}$ may be expressed as

$$
P_{5}=\int_{0}^{\left(|\theta|-\frac{\pi}{12}\right) r} d P_{5}=\frac{\mu_{0} h_{s}}{\pi}\left(\frac{l_{0}+2\left(\frac{\pi}{6}-|\theta|\right) r}{l_{0}+\left(\frac{\pi}{6}-|\theta|\right) r}\right) \ln \left(\frac{2 l_{0}^{2}+\frac{\pi^{2}}{12} r\left(l_{0}+\alpha\right)}{2 l_{0}^{2}+\pi r\left(\frac{\pi}{6}-|\theta|\right)\left(l_{0}+\alpha\right)}\right)
$$

Substituting Equations (13) and (15) into Equation (11) yields

$$
P_{a 1}=\frac{2 \mu_{0} h_{s}}{\pi}\left\{\left(\frac{l_{0}+2\left(|\theta|-\frac{\pi}{12}\right) r}{l_{0}+\left(|\theta|-\frac{\pi}{12}\right) r}\right) \ln \left(\frac{2 l_{0}^{2}+\frac{\pi^{2}}{12} r\left(l_{0}+\alpha\right)}{2 l_{0}^{2}+\pi r\left(|\theta|-\frac{\pi}{12}\right)\left(l_{0}+\alpha\right)}\right)+\left(\frac{l_{0}+2\left(\frac{\pi}{6}-|\theta|\right) r}{l_{0}+\left(\frac{\pi}{6}-|\theta|\right) r}\right) \ln \left(\frac{2 l_{0}^{2}+\frac{\pi^{2}}{12} r\left(l_{0}+\alpha\right)}{2 l_{0}^{2}+\pi r\left(\frac{\pi}{6}-|\theta|\right)\left(l_{0}+\alpha\right)}\right)\right\}
$$

Similarly, $P_{a 2}, P_{a 3}$, and $P_{a 4}$ at the positions of $\frac{\pi}{12} \leq|\theta| \leq \frac{\pi}{8}$ can also be derived following the above method.

\subsubsection{Derivation of Radial Forces in BSRM Part}

Substituting each permeance calculated from the above method into Equation (1), we can get the expressions of the self and mutual inductances. Moreover, it is possible to construct a $4 \times 4$ inductance matrix [L] from the obtained self and mutual inductances, and this $4 \times 4$ inductance matrix $[L]$ is expressed as

$$
[L]=\left[\begin{array}{cccc}
L_{a 1} & M_{a 12} & M_{a 13} & M_{a 14} \\
M_{a 12} & L_{a 2} & M_{a 23} & M_{a 24} \\
M_{a 13} & M_{a 23} & L_{a 3} & M_{a 34} \\
M_{a 14} & M_{a 24} & M_{a 34} & L_{a 4}
\end{array}\right]
$$


The A-phase-stored magnetic energy $W_{a}$ in the BSRM part of a HBSRM can be written using Equation (17) as

$$
W_{a}=\frac{1}{2}\left[\begin{array}{llll}
i_{a 1} & i_{a 2} & i_{a 3} & i_{a 4}
\end{array}\right] \times[L] \times\left[\begin{array}{c}
i_{a 1} \\
i_{a 2} \\
i_{a 3} \\
i_{a 4}
\end{array}\right]
$$

Since the radial force is dependent on the derivative of the stored magnetic energy with respect to the radial displacement of the shaft, the radial forces $F_{\alpha 1}$ and $F_{\beta 1}$ generated by the A-phase in the BSRM part can be written as

$$
\left\{\begin{array}{l}
F_{\alpha 1}=\frac{d W_{a}}{d \alpha}=\frac{1}{8} K_{f 1} N^{2}\left(i_{a 1}+i_{a 2}+i_{a 3}+i_{a 4}\right)\left(i_{a 1}-i_{a 3}\right) \\
F_{\beta 1}=\frac{d W_{a}}{d \beta}=\frac{1}{8} K_{f 1} N^{2}\left(i_{a 1}+i_{a 2}+i_{a 3}+i_{a 4}\right)\left(i_{a 2}-i_{a 4}\right)
\end{array}\right.
$$

where $\beta$ is the radial displacement of the rotor in the $\beta$-axis direction, and $K_{f 1}$ is the coefficient of the radial forces in the BSRM part. It can be expressed as

$$
K_{f 1}=\left\{\begin{array}{l}
\frac{\mu_{0} h_{s} r(\pi-12|\theta|)}{6 l_{0}^{2}}+\frac{4 \mu_{0} h_{s} r|\theta|\left(l_{0}+2|\theta| r\right)}{l_{0}\left(l_{0}+|\theta| r\right)\left(2 l_{0}+\pi|\theta| r\right)}, 0 \leq|\theta| \leq \frac{\pi}{12} \\
\frac{8 \mu_{0} h_{s} r\left(l_{0}+\pi r / 6\right)}{\left(2 l_{0}+\pi^{2} r / 12\right)\left(l_{0}+\pi r / 12\right)}\left(\begin{array}{c}
\frac{\left[l_{0}+2 r(|\theta|-\pi / 12)\right](\pi / 6-|\theta|)}{\left[l_{0}+r(|\theta|-\pi / 12)\right]\left[2 l_{0}+\pi r(|\theta|-\pi / 12)\right]} \\
+\frac{\left[l_{0}+2 r(\pi / 6-|\theta|)\right](|\theta|-\pi / 12)}{\left[l_{0}+r(\pi / 6-|\theta|)\right]\left[2 l_{0}+\pi r(\pi / 6-|\theta|)\right]}
\end{array}\right), \frac{\pi}{12}<|\theta| \leq \frac{\pi}{8}
\end{array}\right.
$$

\subsection{Radial Forces in the AMB Part}

The permeance $P_{a 1}$ of the air gap $a 1$ in the AMB part consists of straight magnetic paths, as shown in Figure 11, and it can be derived as

$$
P_{a 1}=\frac{\mu_{0} h_{c} \pi r\left(l_{0}+\alpha\right)}{12 l_{0}^{2}}
$$

where $h_{c}$ is the stack length of the circular lamination segments. Similarly, $P_{a 2}, P_{a 3}$, and $P_{a 4}$ in the AMB part can also be obtained by the above method.

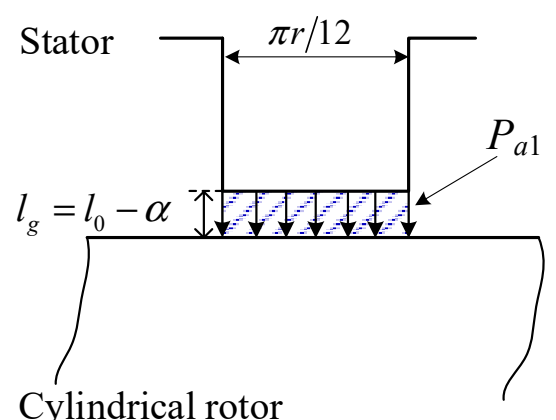

Figure 11. Enlarged flux paths of the air gap $a 1$ in the AMB part.

Similarly, the radial forces $F_{\alpha 2}$ and $F_{\beta 2}$ resulted by the A-phase in the AMB part can be computed from the derivatives of the stored magnetic energy with respect to displacements $\alpha$ and $\beta$, respectively. The radial forces in the AMB part can be written as

$$
\left\{\begin{array}{l}
F_{\alpha 2}=\frac{1}{8} K_{f 2} N^{2}\left(i_{a 1}+i_{a 2}+i_{a 3}+i_{a 4}\right)\left(i_{a 1}-i_{a 3}\right) \\
F_{\beta 2}=\frac{1}{8} K_{f 2} N^{2}\left(i_{a 1}+i_{a 2}+i_{a 3}+i_{a 4}\right)\left(i_{a 2}-i_{a 4}\right)
\end{array}\right.
$$


where $K_{f 2}$ is the coefficient of the radial forces in the AMB part. It can be expressed as

$$
K_{f 2}=\frac{\mu_{0} h_{c} \pi r}{6 l_{0}^{2}}
$$

\subsection{Total Radial Forces of the HBSRM}

The total radial forces of the HBSRM are the sum of both the AMB and BSRM parts. Based on Equations (19), (20), (22), and (23), the total radial forces can be written as

$$
\left\{\begin{array}{l}
F_{\alpha}=F_{\alpha 1}+F_{\alpha 2}=\frac{1}{8} K_{f} N^{2}\left(i_{a 1}+i_{a 2}+i_{a 3}+i_{a 4}\right)\left(i_{a 1}-i_{a 3}\right) \\
F_{\beta}=F_{\beta 1}+F_{\beta 2}=\frac{1}{8} K_{f} N^{2}\left(i_{a 1}+i_{a 2}+i_{a 3}+i_{a 4}\right)\left(i_{a 2}-i_{a 4}\right)
\end{array}\right.
$$

where $K_{f}$ is the total coefficient of the radial forces. It can be expressed as

$$
K_{f}=\left\{\begin{array}{l}
\frac{\mu_{0} h_{c} \pi r}{6 l_{0}^{2}}+\frac{\mu_{0} h_{s} r(\pi-12|\theta|)}{6 l_{0}^{2}}+\frac{4 \mu_{0} h_{s} r|\theta|\left(l_{0}+2|\theta| r\right)}{l_{0}\left(l_{0}+|\theta| r\right)\left(2 l_{0}+\pi|\theta| r\right)}, 0 \leq|\theta| \leq \frac{\pi}{12} \\
\frac{\mu_{0} h_{c} \pi r}{6 l_{0}^{2}}+\frac{8 \mu_{0} h_{s} r\left(l_{0}+\pi r / 6\right)}{\left(2 l_{0}+\pi^{2} r / 12\right)\left(l_{0}+\pi r / 12\right)}\left(\begin{array}{c}
\frac{\left[l_{0}+2 r(|\theta|-\pi / 12)\right](\pi / 6-|\theta|)}{\left[l_{0}+r(|\theta|-\pi / 12)\right]\left[2 l_{0}+\pi r(|\theta|-\pi / 12)\right]} \\
+\frac{\left[l_{0}+2 r(\pi / 6-\mid \theta) \mid(|\theta|-\pi / 12)\right.}{\left[l_{0}+r(\pi / 6-|\theta|)\right]\left[2 l_{0}+\pi r(\pi / 6-|\theta|)\right]}
\end{array}\right), \frac{\pi}{12}<|\theta| \leq \frac{\pi}{8}
\end{array}\right.
$$

\subsection{Torques of the HBSRM}

The A-phase torque $T_{a}$ can be obtained by the derivatives of the stored magnetic energy $W_{a}$ with respect to the rotor angular positon $\theta$. Since the AMB part generates no torque, the total torques of HBSRM are equal to that produced by the BSRM part. Therefore, the A-phase torque expression of HBSRM can be written as

$$
T_{a}=\frac{d W_{a}}{d \theta}=\frac{1}{8} J_{t} N^{2}\left[\left(i_{a 1}+i_{a 2}+i_{a 3}+i_{a 4}\right)^{2}+2\left(i_{a 1}-i_{a 3}\right)^{2}+2\left(i_{a 2}-i_{a 2}\right)^{2}\right]
$$

where $J_{t}$ is the coefficient of the torques in the BSRM part. It can be expressed as

$$
J_{t}=\left\{\begin{array}{l}
2 \mu_{0} h_{s} r\left(\frac{l_{0}-2 r(\theta+\pi / 12)}{\left[l_{0}-r(\theta+\pi / 12)\right]\left[2 l_{0}-\pi r(\theta+\pi / 12)\right]}-\frac{l_{0}+2 r(\theta+\pi / 6)}{\left[l_{0}+r(\theta+\pi / 6)\right]\left[2 l_{0}+\pi r(\theta+\pi / 6)\right]}\right),-\frac{\pi}{8} \leq \theta<-\frac{\pi}{12} \\
\mu_{0} h_{s} r\left(\frac{1}{l_{0}}-\frac{2\left(l_{0}-2 \theta r\right)}{\left(l_{0}-\theta r\right)\left(2 l_{0}-\pi \theta r\right)}\right),-\frac{\pi}{12} \leq \theta<0 \\
\mu_{0} h_{s} r\left(-\frac{1}{l_{0}}+\frac{2\left(l_{0}+2 \theta r\right)}{\left(l_{0}+\theta r\right)\left(2 l_{0}+\pi \theta r\right)}\right), 0 \leq \theta \leq \frac{\pi}{12} \\
2 \mu_{0} h_{s} r\left(-\frac{l_{0}+2 r(\theta-\pi / 12)}{\left[l_{0}+r(\theta-\pi / 12)\right]\left[2 l_{0}+\pi r(\theta-\pi / 12)\right]}+\frac{l_{0}+2 r(\pi / 6-\theta)}{\left[l_{0}+r(\pi / 6-\theta)\right]\left[2 l_{0}+\pi r(\pi / 6-\theta)\right]}\right), \frac{\pi}{12}<\theta \leq \frac{\pi}{8}
\end{array}\right.
$$

\subsection{Mathematical Model of the Double-Winding Structure}

In essence, a BSRM uses the asymmetry of air gap magnetic fluxes in the radial direction to generate suspended forces. These asymmetric air gap magnetic fluxes can be generated by single-winding or double-winding excitations. Figure 12 shows the A-phase winding configuration and magnetic flux lines by finite element analysis in a 12/8-pole double-winding BSRM. The motor main winding $N_{m a}$ consists of four coils connected in a series. On the other hand, the radial force windings $N_{s a 1}$ and $N_{s a 1}$ consist of two coils each. The B-phase winding configuration is situated on the one-third rotational position of the A-phase. The C-phase winding configuration is situated on the two-thirds rotational position of the A-phase. 


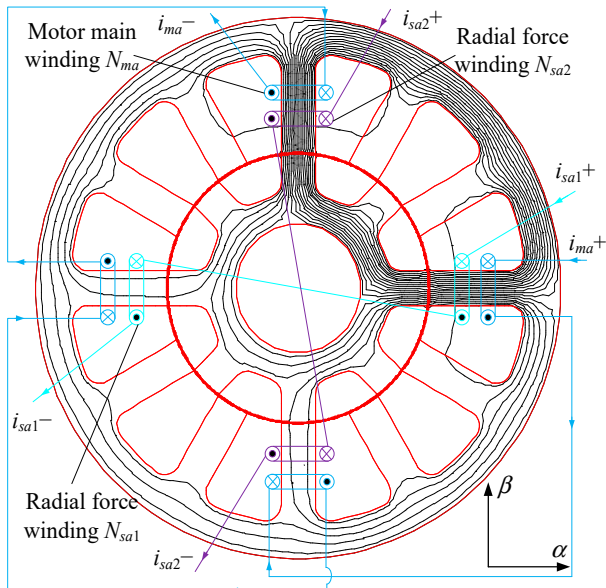

Figure 12. A-phase winding configuration and magnetic flux lines in a 12/8-pole doublewinding BSRM.

Based on the principle of magnetomotive force balance, the equivalent relations between single-winding and double-winding structures can be expressed as

$$
\left\{\begin{array}{l}
N i_{a 1}=N_{m} i_{m a}+N_{b} i_{s a 1} \\
N i_{a 2}=N_{m} i_{m a}+N_{b} i_{s a 2} \\
N i_{a 3}=N_{m} i_{m a}-N_{b} i_{s a 1} \\
N i_{a 4}=N_{m} i_{m a}-N_{b} i_{s a 2}
\end{array}\right.
$$

where $N_{m}$ is the number of turns of the motor main winding, $N_{b}$ is the number of turns of radial force winding, $i_{m a}$ is the current in the motor main winding $N_{m a}, i_{s a 1}$ is the current in the radial force winding $N_{s a 1}$, and $i_{s a 2}$ is the current in the radial force winding $N_{s a 1}$.

Substituting Equation (28) into Equations (24) and (26), the radial force and torque expressions of the HBSRM with double-winding configurations on each stator tooth can be written as

$$
\begin{gathered}
\left\{\begin{array}{l}
F_{\alpha}=K_{f} N_{m} N_{b} i_{m a} i_{s a 1} \\
F_{\beta}=K_{f} N_{m} N_{b} i_{m a} i_{s a 2}
\end{array}\right. \\
T_{a}=J_{t}\left(2 N_{m}^{2} i_{m a}^{2}+N_{b}^{2} i_{s a 1}^{2}+N_{b}^{2} i_{s a 2}^{2}\right)
\end{gathered}
$$

It can be seen from Equations (29) and (30) that the expressions are consistent with those in References $[7,8]$. Therefore, the radial force and torque coefficients derived from the single-winding structure can be directly applied to the double-winding BSRM.

\section{Model Analysis}

Figure 13 shows the radial force and torque coefficients obtained by the classical model and novel model of the BSRM part based on the parameters in Table 1. It is known from Figure 13 that the torque coefficient derived from the classical method is not zero at the position of $\theta=0$, and the radial force coefficient obtained by the classical method is discontinuous at the aligned position. However, these problems can be solved by the new model presented in this paper, because the torque is proportional to the derivative of the inductance with respect to the rotor angular position $\theta$. Moreover, if the derivative of the gap permeance at the aligned position is continuous, the torque is also continuous at this position. The continuity of the new model is analyzed as follows: 


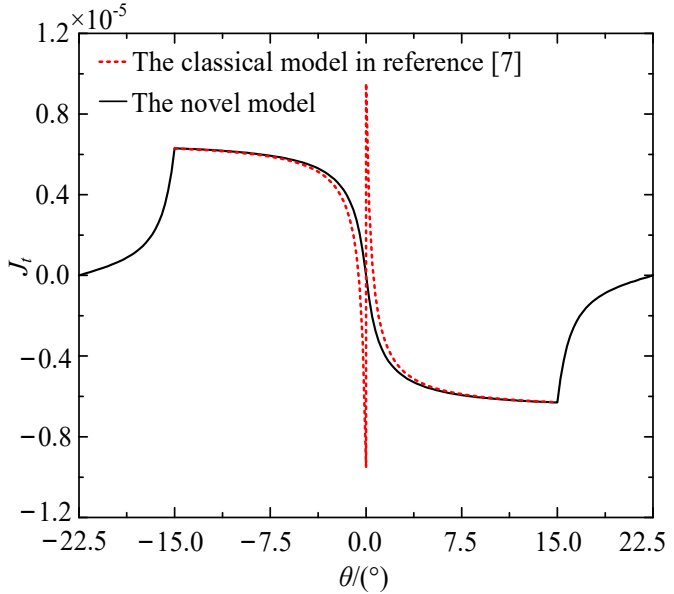

(a)

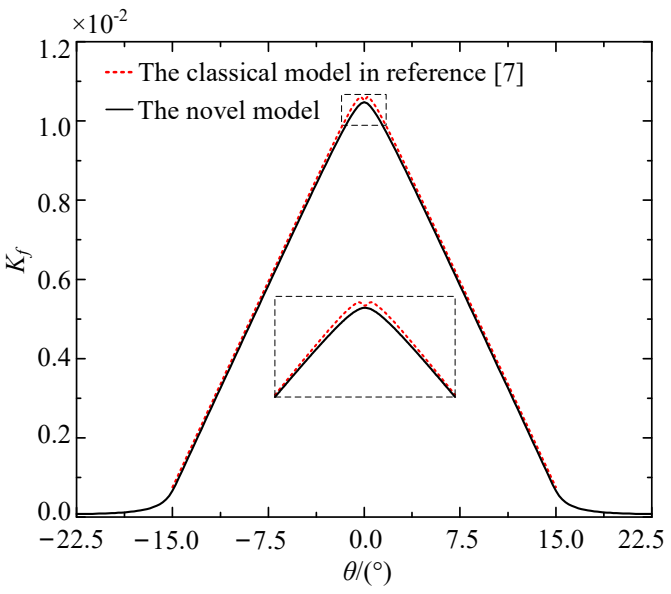

(b)

Figure 13. Comparison of the radial force and torque coefficients in the BSRM part. (a) Torque coefficient $J_{t}$. (b) Radial force coefficient $K_{f}$.

When the rotor rotates from the positive torque period of $\theta<0$ to the aligned position, the derivative of $P_{a 1}$ at the aligned position can be expressed as

$$
\lim _{\theta \rightarrow 0-} \frac{d P_{a 1}}{d \theta}=\lim _{\theta \rightarrow 0-}\left(\frac{d P_{1}}{d \theta}+2 \frac{d P_{2}}{d \theta}\right)=\frac{\mu_{0} h_{s} r}{l_{0}}-\frac{\mu_{0} h_{s} r}{l_{0}}=0
$$

Similarly, if the rotor oppositely rotates from the negative torque period of $\theta>0$ to the aligned position, the derivative of $P_{a 1}$ at the aligned position is written as

$$
\lim _{\theta \rightarrow 0+} \frac{d P_{a 1}}{d \theta}=\lim _{\theta \rightarrow 0+}\left(\frac{d P_{1}}{d \theta}+2 \frac{d P_{2}}{d \theta}\right)=-\frac{\mu_{0} h_{s} r}{l_{0}}+\frac{\mu_{0} h_{s} r}{l_{0}}=0
$$

Moreover, the derivative of $P_{a 1}$ at the unaligned positions of $\theta= \pm \pi / 8$ can be written as

$$
\left\{\begin{array}{c}
\lim _{\theta \rightarrow\left(-\frac{\pi}{8}\right)+} \frac{d P_{a 1}}{d \theta}=\lim _{\theta \rightarrow\left(-\frac{\pi}{8}\right)+}\left(2 \frac{d P_{4}}{d \theta}+2 \frac{d P_{5}}{d \theta}\right)=\frac{2 \mu_{0} h_{s} r\left(l_{0}+\frac{\pi r}{12}\right)}{\left(l_{0}+\frac{\pi r}{24}\right)\left(2 l_{0}+\frac{\pi^{2} r}{24}\right)}-\frac{2 \mu_{0} h_{s} r\left(l_{0}+\frac{\pi r}{12}\right)}{\left(l_{0}+\frac{\pi r}{24}\right)\left(2 l_{0}+\frac{\pi^{2} r}{24}\right)}=0 \\
\lim _{\theta \rightarrow \frac{\pi}{8}-} \frac{d P_{a 1}}{d \theta}=\lim _{\theta \rightarrow \frac{\pi}{8}-}\left(2 \frac{d P_{4}}{d \theta}+2 \frac{d P_{5}}{d \theta}\right)=-\frac{2 \mu_{0} h_{s} r\left(l_{0}+\frac{\pi r}{12}\right)}{\left(l_{0}+\frac{\pi r}{24}\right)\left(2 l_{0}+\frac{\pi^{2} r}{24}\right)}+\frac{2 \mu_{0} h_{s} r\left(l_{0}+\frac{\pi r}{12}\right)}{\left(l_{0}+\frac{\pi r}{24}\right)\left(2 l_{0}+\frac{\pi^{2} r}{24}\right)}=0
\end{array}\right.
$$

In addition, the torque coefficient at the positions of $\theta= \pm \pi / 12$ may be expressed as

$$
\left\{\begin{array}{l}
\left.J_{t}\right|_{\theta=\frac{\pi}{12}}=\left.\left(\frac{d P_{1}}{d \theta}+2 \frac{d P_{2}}{d \theta}\right)\right|_{\theta=\frac{\pi}{12}}=\left.\left(2 \frac{d P_{4}}{d \theta}+2 \frac{d P_{5}}{d \theta}\right)\right|_{\theta=\frac{\pi}{12}}=\mu_{0} h_{s} r\left(-\frac{1}{l_{0}}+\frac{2\left(l_{0}+\frac{\pi r}{6}\right)}{\left(l_{0}+\frac{\pi r}{12}\right)\left(2 l_{0}+\frac{\pi^{2} r}{12}\right)}\right) \\
\left.J_{t}\right|_{\theta=-\frac{\pi}{12}}=\left.\left(\frac{d P_{1}}{d \theta}+2 \frac{d P_{2}}{d \theta}\right)\right|_{\theta=-\frac{\pi}{12}}=\left.\left(2 \frac{d P_{4}}{d \theta}+2 \frac{d P_{5}}{d \theta}\right)\right|_{\theta=-\frac{\pi}{12}}=\mu_{0} h_{s} r\left(\frac{1}{l_{0}}-\frac{2\left(l_{0}+\frac{\pi r}{6}\right)}{\left(l_{0}+\frac{\pi r}{12}\right)\left(2 l_{0}+\frac{\pi^{2} r}{12}\right)}\right)
\end{array}\right.
$$

In an SRM, the torques at the aligned and unaligned positions invariably become zero. It can be seen from Equations (31)-(33) that the torque expressions proposed in the paper at the aligned and unaligned positions are zero, and the torques at these positions are continuous. Otherwise, it is noted from Equation (34) that the novel model also ensures the consistency of the torque, although it is derived from different regions and assumed magnetic paths. Moreover, the continuity of the radial force formulas at all the rotor angular positons can also be verified using a similar method.

Figure 14 shows the calculated results of the radial forces and torques using the parameters of the prototype shown in Table 1. A two-dimensional finite element analysis for the A-phase of HBSRM was carried out in this paper. In the radial force calculations, the currents in four windings of the A-phase are, respectively, set as follows: $i_{a 1}=4 \mathrm{~A}, i_{a 2}=2 \mathrm{~A}$, 
$i_{a 3}=0$, and $i_{a 4}=2 \mathrm{~A}$. In the torque simulation, the A-phase current is set to $i_{a 1}=i_{a 2}=i_{a 3}=$ $i_{a 4}=4 \mathrm{~A}$. It can be seen from Figure 14 that the novel full-period mathematical model has good accuracy, and both the radial force and torque are continuous at all the positions of each rotor period.

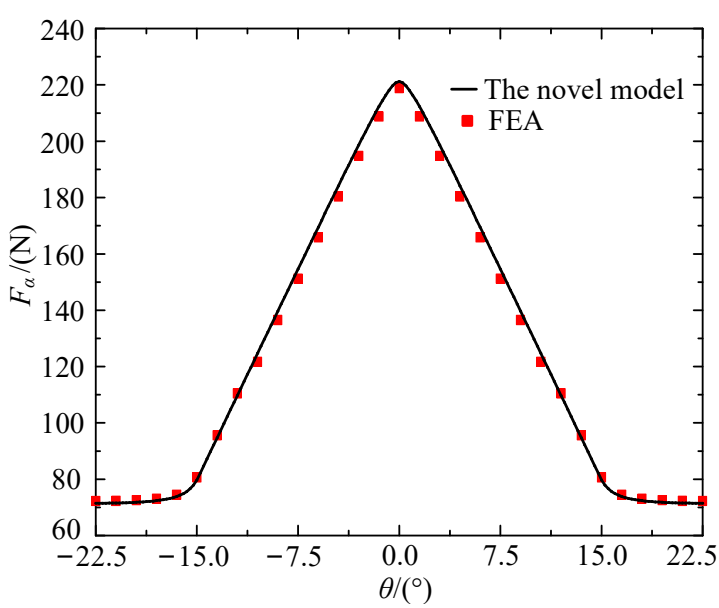

(a)

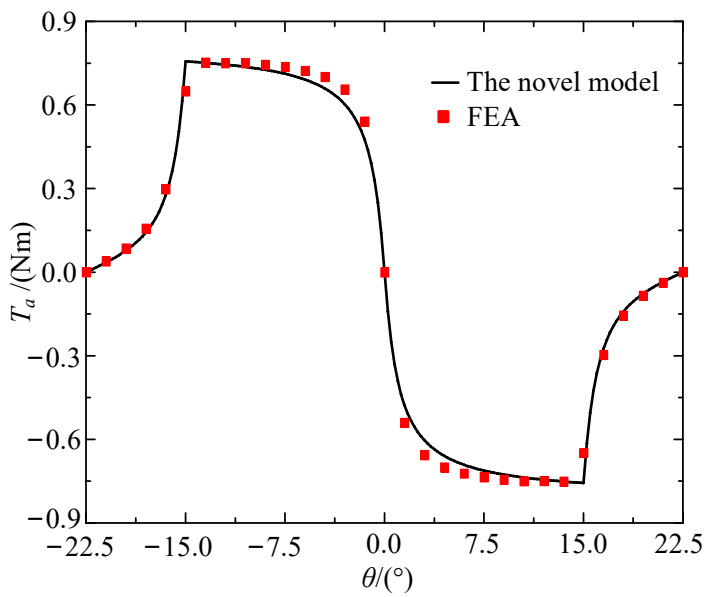

(b)

Figure 14. Comparison of the radial forces and torques. (a) Radial forces. (b) Torques.

The radial force results in Figure 14 further show that the HBSRM has the ability to suspend the shaft in any rotor angular position of each phase. Moreover, this structure can solve the problem that is restricting the performance improvement of the traditional BSRM due to conflicts between the effective output regions of the torque and radial force. Therefore, based on this new model presented in this paper, the radial force excitation interval of each phase and more flexible control algorithm for the HBSRM can be further optimized to improve the motor's output performance.

\section{Conclusions}

A 12/8-pole BSRM with a hybrid rotor composed of circular and scalloped lamination segments was discussed in this paper. The cylindrical rotor segment of the HBSRM was used to generate additional radial forces, which could solve the problem that the radial force of a traditional BSRM near the unaligned positions of each phase is insufficient to levitate a rotor shaft. In order to simplify the asymmetric magnetic field of the 12/8-pole HBSRM, this motor was regarded as a 12/8-pole BSRM and a 12-pole AMB, and then, the equivalent magnetic circuits of both parts were established for the derivation of the radial force and torque formulas. Moreover, a novel full-period mathematical model for the 12/8-pole HBSRM was derived, which was applicable to any rotor angular position of each phase. Compared with the classical model of the traditional BSRM, the new model can ensure the continuity of the radial force and torque at all the rotor positions, and the excellent characteristics of this model were confirmed by a finite element analysis. In addition, the full-period model for the HBSRM was quite helpful in optimizing this motor's radial force excitation interval of each phase and the control algorithm for the improvement of the output performance.

Author Contributions: Conceptualization, Z.L.; methodology, Z.L. and M.C.; validation, Z.L., M.C., Y.Y. and C.L.; writing—original draft preparation, Z.L. and H.G.; writing—review and editing, Z.L. and M.C.; and funding acquisition, Z.L. All authors have read and agreed to the published version of the manuscript.

Funding: This research was supported in part by the National Natural Science Foundation of China under grant 51607097, in part by the Opening Foundation of Key Laboratory of Special Machine and High Voltage Apparatus under grant KFKT202102, and in part by the Scientific Foundation of Nanjing University of Posts and Telecommunications under grant NY220010. 
Institutional Review Board Statement: Not applicable.

Informed Consent Statement: Not applicable.

Data Availability Statement: The raw data supporting the conclusions of this article will be made available by the authors without undue reservation.

Conflicts of Interest: The authors declare no conflict of interest.

\section{References}

1. Gerada, D.; Mebarki, A.; Brown, N.L.; Gerada, C.; Cavagnino, A.; Boglietti, A. High-speed electrical machines: Technologies, trends, and developments. IEEE Trans. Ind. Electron. 2014, 61, 2946-2959. [CrossRef]

2. Chen, H.; Yan, W.; Gu, J.; Sun, M. Multiobjective optimization design of a switched reluctance motor for low-speed electric vehicles with a taguchi-cso algorithm. IEEE/ASME Trans. Mechatron. 2018, 23, 1762-1774. [CrossRef]

3. Gan, C.; Chen, Y.; Cui, X.; Sun, J.; Ni, K.; Qu, R. Investigation of rotor strength and rotor dynamics for high-speed high-power switched reluctance machines. IET Electr. Power Appl. 2020, 14, 1624-1630. [CrossRef]

4. Zhang, J.W.; Wang, H.H.; Chen, L.; Tan, C.; Wang, Y. Multi-objective optimal design of bearingless switched reluctance motor based on multi-objective genetic particle swarm optimizer. IEEE Trans. Magn. 2018, 54, 8100113. [CrossRef]

5. Sun, Y.K.; Yang, F.; Yuan, Y.; Yu, F.Y.; Xiang, Q.W.; Zhu, Z.Y. Analysis of a hybrid double stator bearingless switched reluctance motor. Electron. Lett. 2018, 54, 1397-1399. [CrossRef]

6. Wang, H.J.; Liu, F.X. Design consideration and characteristic investigation of modular permanent magnet bearingless switched reluctance motor. IEEE Trans. Ind. Electron. 2020, 67, 4326-4337. [CrossRef]

7. Takemoto, M.; Shimada, K.; Chiba, A.; Fukao, T. A design and characteristics of switched reluctance type bearingless motors. In Proceedings of the 4th International Symposium on Magnetic Suspension Technology, NASA/CP-1998-207654, Gifu, Japan, 30 October-1 November 1997; pp. 49-63.

8. Takemoto, M.; Chiba, A.; Fukao, T. A new control method of bearingless switched reluctance motors using square-wave currents. In Proceedings of the 2000 IEEE Power Engineering Society Winter Meeting, Singapore, 23-27 January 2000; pp. 375-380.

9. Takemoto, M.; Chiba, A.; Fukao, T. A method of determining advanced angle of square-wave currents in a bearingless switched motor. IEEE Trans. Ind. Applicat. 2001, 37, 1702-1709. [CrossRef]

10. Takemoto, M.; Suzuki, H.; Chiba, A.; Fukao, T.; Azizur Rahman, M. Improved analysis of a bearingless switched reluctance motor. IEEE Trans. Ind. Applicat. 2001, 37, 26-34. [CrossRef]

11. Choi, B.B.; Siebert, M. A bearingless switched reluctance motor for high specific power applications. In Proceedings of the 42nd AIAA/ASME/SAE/ASEE Joint Propulsion Conference \& Exhibit, Sacramento, CA, USA, 9-12 July 2006; pp. 4832-4842.

12. Chen, L.; Hofmann, W. Speed regulation technique of one bearingless $8 / 6$ switched reluctance motor with simpler single winding structure. IEEE Trans. Ind. Electron. 2012, 59, 2592-2600. [CrossRef]

13. Cao, X.; Yang, H.; Zhang, L.; Deng, Z.Q. Compensation strategy of levitation forces for single-winding bearingless switched reluctance motor with one winding total short circuited. IEEE Trans. Ind. Electron. 2016, 63, 5534-5546. [CrossRef]

14. Cao, X.; Zhou, J.X.; Liu, C.Y.; Deng, Z.Q. Advanced control method for a single-winding bearingless switched reluctance motor to reduce torque ripple and radial displacement. IEEE Trans. Energy Convers. 2017, 32, 1533-1543. [CrossRef]

15. Wang, H.J.; Liu, J.F.; Bao, J.F.; Xue, B.K. A novel bearingless switched reluctance motor with a biased permanent magnet. IEEE Trans. Ind. Electron. 2014, 61, 6947-6955. [CrossRef]

16. Takemoto, M.; Chiba, A.; Akagi, H.; Fukao, T. Radial force and torque of a bearingless switched reluctance motor operating in a region of magnetic saturation. IEEE Trans. Ind. Applicat. 2004, 40, 103-112. [CrossRef] 